\title{
Staphylococcus aureus biofilm formation at the physiologic glucose concentration depends on the $S$. aureus lineage Sander Croes ${ }^{1,2}$, Ruud H Deurenberg ${ }^{1}$, Marie-Louise L Boumans ${ }^{1}$, Patrick S Beisser ${ }^{1}$, Cees Neef ${ }^{2}$ and Ellen E Stobberingh*1
}

\author{
Address: ${ }^{1}$ Department of Medical Microbiology, Maastricht University Medical Center, Maastricht, The Netherlands and ${ }^{2}$ Department of Clinical \\ Pharmacy and Toxicology, Maastricht University Medical Center, Maastricht, The Netherlands \\ Email: Sander Croes - S.Croes@mumc.nl; Ruud H Deurenberg - Ruud.deurenberg@mumc.nl; Marie- \\ Louise L Boumans - m.boumans@mumc.nl; Patrick S Beisser - p.beisser@mumc.nl; Cees Neef - C.Neef@mumc.nl; \\ Ellen E Stobberingh* - e.stobberingh@mumc.nl \\ * Corresponding author
}

Published: 28 October 2009

BMC Microbiology 2009, 9:229 doi:10.1 186/147|-2180-9-229

This article is available from: http://www.biomedcentral.com/l47I-2/80/9/229

(c) 2009 Croes et al; licensee BioMed Central Ltd.

This is an Open Access article distributed under the terms of the Creative Commons Attribution License (http://creativecommons.org/licenses/by/2.0), which permits unrestricted use, distribution, and reproduction in any medium, provided the original work is properly cited.

\begin{abstract}
Background: Since bacteria embedded in biofilms are far more difficult to eradicate than planktonic infections, it would be useful to know whether certain Staphylococcus aureus lineages are especially involved in strong biofilm formation. For this reason, in vitro biofilm formation of 228 clinical S. aureus isolates of distinct clonal lineages was investigated.
\end{abstract}

Results: At $0.1 \%$ glucose, more than $60 \%$ of the $S$. aureus strains associated with multilocus sequence typing (MLST) clonal complex (CC)8 produced large amounts of biomass, compared to $0-7 \%$ for various other clonal lineages. Additionally, S. aureus bloodstream isolates associated with MLST CC8 and CC7 had similar biofilm forming capacities as their commensal counterparts. Furthermore, strong biofilm formation could not be attributed to a specific accessory gene regulator (agr) genotype, as suggested previously. The agr genotypes were strictly associated with the clonal lineages. Moreover, strong biofilm formation was not related to slime formation. Congo red agar (CRA) screening is therefore not useful as a qualitative screening method for biofilm formation.

Conclusion: The adherence to polystyrene surfaces under physiologic glucose concentration $(0.1 \%)$ was dependent on the clonal lineage. Strains associated with MLST CC8 were markedly more often classified as strong biofilm former at glucose concentrations of $0 \%, 0.1 \%$ and $0.25 \%$.

The present study reveals that the MLST CC8 associated genetic background was a predisposing factor for strong biofilm formation in vitro, under all tested glucose concentrations.

\section{Background}

One of the defense mechanisms of Staphylococcus aureus is the capacity to form biofilms. Bacteria embedded in biofilms are often difficult to eradicate with standard antibiotic regimens and inherently resistant to host immune responses [1,2]. As a result, treatment of many chronic $S$. aureus biofilm related infections, including endocarditis, osteomyelitis and indwelling medical device infections is hindered [3]. Biofilm formation is a multistep process, starting with transient adherence to a surface. Subse- 
quently, specific bacterial adhesins, referred to as microbial surface components recognizing adhesive matrix molecules (MSCRAMMS) promote the actual attachment [4]. Next, during the accumulation phase, bacteria stick to each other and production of extracellular polymeric substances (EPS) and/or incorporation of host derived components, such as platelets, takes place, resulting in a mature biofilm. In circumstances of nutrient deprivation, or under heavy shear forces, detachment of bacteria appears through autonomous formation of autoinducing peptides (AIP) [5], with release and dispersal of bacteria as a consequence. It has been shown that expression of the accessory gene regulator (agr) locus, encoding a quorumsensing system, results in expression of surfactant-like molecules, such as $\delta$-toxin [6], contributing to the detachment.

Essential for biofilm development in S. aureus is the regulatory genetic locus staphylococcal accessory regulator ( sarA), which controls the intracellular adhesin (ica) operon and agr regulated pathways [7]. It has been suggested that biofilm formation in methicillin-resistant $S$. aureus (MRSA) is predominantly regulated by surface adhesins, which are repressed under agr expression, while biofilm formation in methicillin-susceptible $S$. aureus (MSSA) is more dependent on cell to cell adhesion by the production of icaADBC-encoded polysaccharide intercellular adhesin (PIA), also referred as poly- $N$-acetylglucosamine (PNAG) or slime [8]. However a clear role for the ica locus of S. aureus is not as evident as that of Staphylococcus epidermidis [9].

In general, the presence of glucose represses the agr system through the generation of a low $\mathrm{pH}[10,11]$. So far, biofilm development in physiologic glucose-supplemented medium $(1 \mathrm{~g} / \mathrm{L})$, corresponding to normal blood glucose levels [12], has not been investigated. Biofilm formation often occurs on medical devices, like catheters and heart valves, which are in direct contact with normal (floating) blood. Furthermore, since it has been shown that the regulatory pathways for biofilm formation vary between strains [8], the question arose whether these strain-tostrain differences could be attributed to different clonal lineages.

The aim of the present study was to examine the contribution of the genetic background of both MRSA and MSSA to biofilm formation under physiologic glucose concentration. MRSA associated with the five major multilocus sequence typing (MLST) clonal complexes (CCs), i.e. CC5, CC8, CC22, CC30 and CC45 [13] and MSSA with the same MLST CCs, and also CC1, were included in this study, since it has been suggested that these lineages possess the ability to become MRSA [14]. The results were compared with those obtained with lineages normally not related to MRSA, i.e. CC7, CC12, CC15, CC25 and CC121 [15]. Furthermore, the aim was to evaluate whether slime production is indicative for strong biofilm formation in $S$. aureus.

\section{Results \\ Characterization of the genetic background}

The spa types and associated MLST CCs of all tested strains are shown in Table 1 . The results of spa typing/BURP and MLST were in accordance for a representative set of 16 strains of each major spa type and associated MLST CC.

\section{Phenotypic detection of slime producing ability onto Congo red agar}

The different Congo red agar (CRA) screening methods described in the literature were evaluated [16-18]. The choice of the agar medium, either brain heart infusion or trypticase soy, did not influence the morphology. The majority of S. aureus strains (91\%) displayed colonies with a normal morphology (smooth round colonies), indicating that most strains were low-slime producers. Without sucrose, all colonies were colored (bright) red to bordeaux red, irrespective of the agar medium used. Addition of sucrose to both agar media resulted in more dark colonies and made the dry crystalline morphology harder to recognize. With sucrose, all colonies on brain heart infusion agar with Congo red were colored red to bordeaux red, while strains on trypticase soy agar with Congo red displayed mostly purple to black colonies. Nuances in color were not corresponding to differences in morphology.

MSSA strains showed more often a deviant, dry crystalline (rough) morphology (slime producing positive) than MRSA isolates, $14 \%$ (22 of 156) and 0\%, respectively. A significant distinction in slime formation was observed between MRSA and MSSA with MSSA associated MLST CCs, i.e. CC7, CC12, CC15, CC25 and CC121, and with MRSA associated MLST CCs, i.e. CC1, CC5, CC8, CC22, CC30 and CC45 $(P<0.01)$, as shown in Figure 1a. MSSA associated with MLST CC121 had the highest prevalence of a deviant morphology, 67\% (10 of 15) (Figure 1b).

\section{Detection of biofilm biomass with crystal violet staining} Under physiologic glucose $(0.1 \%)$ concentration, $13 \%$ ( $n$ $=30$ ) of all strains formed a strong biofilm and all these strains were MRSA or had a MRSA associated MLST CC. MRSA and MSSA with MRSA associated MLST CCs, i.e. CC1, CC5, CC8, CC22, CC30 and CC45, were significantly more capable than MSSA with MSSA associated MLST CCs, i.e. CC7, CC12, CC15, CC25 and CC121, to form strong biofilms in the presence of $0.1 \%$ glucose $(P<$ 0.01 ), but not at glucose concentrations of $0.25 \%$ and $0.5 \%$ (Figure 2). The higher the glucose concentration, the more strains produced biofilm above the $A_{590}$ threshold 
Table I: Distribution of spa types and associated MLST CCs among S. aureus strains included in this study

\begin{tabular}{|c|c|c|c|c|c|c|}
\hline $\begin{array}{l}\text { associated } \\
\text { MLST CC }\end{array}$ & ST & $\begin{array}{l}\text { No. of MRSA } \\
\text { strains }\end{array}$ & $\begin{array}{l}\text { No. of MSSA } \\
\text { strains }\end{array}$ & agr genotype & $\begin{array}{c}\text { spa types MRSA } \\
\text { strains } \\
\text { (No.) }\end{array}$ & $\begin{array}{c}\text { spa types MSSA } \\
\text { strains } \\
\text { (No.) }\end{array}$ \\
\hline 1 & STI & NA\# & 16 & III & $N A^{\#}$ & tI $27(15), \operatorname{tl} 787$ \\
\hline 5 & ST5/ST5 & 15 & 15 & II & $\begin{array}{l}\text { t002 (4), t003, t04I, } \\
\text { t045, t447 (8) }\end{array}$ & $\begin{array}{l}\text { t002 (I2), tl79, t3III, } \\
\text { t22I2 }\end{array}$ \\
\hline 8 & ST8/STI4IIa & 26 & 15 & 1 & $\begin{array}{l}\text { t008 (12), t052 (6), } \\
\text { t064, t068 (5), t303, } \\
\text { t622 }\end{array}$ & $\begin{array}{l}\text { t008a (10), t } 190, \mathrm{t} 648 \\
\text { t70I (2), t204I }\end{array}$ \\
\hline 22 & ST22/ST22 & 10 & 15 & 1 & $\mathbf{t} 223(10)$ & $\begin{array}{l}\text { t005 (9), t223, t474, t790, } \\
\text { tl } 433, \mathrm{t} \mid 629, \mathrm{t} 268 \mathrm{I}\end{array}$ \\
\hline 30 & ST36/ST7I $4^{b}$ & 10 & 15 & III & $\begin{array}{l}\text { t0I2 (7), t253 (2), } \\
\text { t1820 }\end{array}$ & $\begin{array}{l}\mathrm{t} 012(2), \mathrm{t} 02 \mathrm{I}^{\mathrm{b}}(4), \mathrm{t} 238, \\
\mathrm{t} 300, \mathrm{t} 3 \mid 8(2), \mathrm{t} 438, \\
\mathrm{t} \mathrm{I}|30, \mathrm{t}| 504, \mathrm{t} 2572, \\
\mathrm{t} 2854\end{array}$ \\
\hline 45 & ST45/ST45 & 11 & 15 & I & $\begin{array}{l}\text { t038 (8), t445 (2), } \\
\text { t740 }\end{array}$ & $\begin{array}{l}\mathrm{t} 015 \text { (2), t026, t050, t065, } \\
\mathrm{t} 102, \mathrm{t} 230(3), \mathrm{t} 583, \mathrm{t} 589, \\
\mathrm{t} 620(2), \mathrm{t} 772(2)\end{array}$ \\
\hline 7 & ST7 & - & 15 & 1 & - & t09l (I5) \\
\hline 12 & STI2 & - & 10 & II & - & $\begin{array}{l}\text { t060, tI56 (2), t } 160(5) \\
\text { t2I3, t77। }\end{array}$ \\
\hline 15 & STI5 & - & 15 & ॥ & - & $\begin{array}{l}\text { t084 (II), t085, t49I (2), } \\
\text { tl716 }\end{array}$ \\
\hline 25 & ST25 & - & 10 & I & - & $\begin{array}{l}\text { t078 (4), t08I, t087, t258, } \\
\text { t353, tl67।, t } 1898\end{array}$ \\
\hline 121 & ST720c & - & 15 & IV & - & $\begin{array}{l}\mathrm{t} 159(2), \mathrm{t}|7| \mathrm{c}(4), \mathrm{t} 284 \\
\mathrm{t} 408(4), \mathrm{t} 645(2), \mathrm{t} 659 \\
\mathrm{t} 2213\end{array}$ \\
\hline Total & & 72 & 156 & & & \\
\hline
\end{tabular}

\# not available

Boldface indicates spa types on which multilocus sequence typing analysis was performed (ST, sequence type).

a The strain spa typed as t008 had STI4II, a double locus variant of ST8 at the gmk and tpi locus.

b The strain spa typed as t02I had ST714, a single locus variant of ST30 at the arcc locus.

c The strain spa typed as tI7I had ST720, a single locus variant of STI2I at the yqil locus.

value and were consequently classified as strong biofilm former. At glucose concentrations of $0.25 \%$ and $0.5 \%$, the amount of biomass of the biofilms of strong biofilm forming strains was still significantly more for MRSA compared to MSSA irrespective of the MLST CCs $(P<0.01)$ (Figure 3). Of all strains classified as strong biofilm producers, MRSA and MSSA associated with MLST CC8 produced the most biomass under all tested glucose concentrations (Figure $4 \mathrm{a}$ and $4 \mathrm{~b}$ ). Strains defined as strong biofilm formers and associated with MLST CC5, CC25 and CC30 approached approximately the same level of biomass at the following glucose concentrations, i.e. CC5 at $0.25 \%$, CC 25 at $0.5 \%$ and CC 30 at $0.5 \%$ glucose, respectively.

The main contributors to the higher prevalence of MRSA and MSSA with MRSA associated MLST CCs to produce strong biofilms at $0.1 \%$ glucose were MLST CC 8 isolates, approximately $60 \%$ (26 of 41), (Figure 4c), especially with a tendency towards MRSA (Figure 4d).

Additionally, blood stream isolates of MSSA associated with MLST CC8 and MLST CC7 were included in the study, to address the question whether the isolation site is an (additional) predisposing factor for strong biofilm formation. MSSA associated with MLST CC7 are one of the main clonal lineages among blood stream isolates in our hospital [19]. No differences in the ability to produce strong biofilms were observed between bloodstream isolates and isolates of commensal origin among MSSA associated with MLST CC8 and CC7 (Figure 5a and 5b). Furthermore, no significant differences in slime-forming ability were observed (Figure 5c).

\section{Correlation between slime formation and development of biofilm biomass}

In order to investigate whether slime production is indicative for strong biofilm formation, the correlation between these two characteristics was addressed. Phenotypic detection of slime production on CRA was not related to the quantitative detection of strong biofilms, measured by crystal violet staining, which was used as a gold standard. The sensitivity and specificity of the CRA method for $S$. aureus was approximately $9 \%$ and $90 \%$, respectively (Table 2). Only a part of the slime producing strains surpassed the $A_{590}$ threshold value for strong bio- 

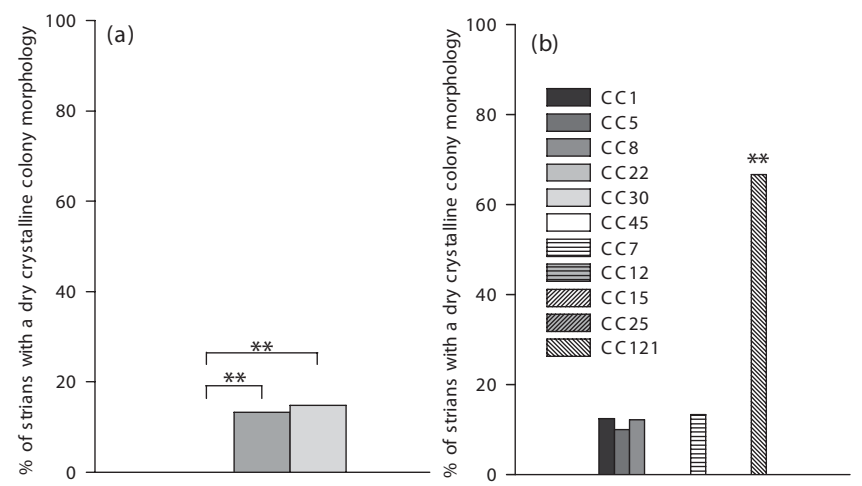

Figure I

Congo Red Agar screening of $S$. aureus isolates. CRA screening for $S$. aureus with a dry crystalline colony morphology, which was considered indicative for slime formation. (a) The black bar (not visible, $0 \%$ ) represents MRSA $(n=72)$, the dark grey bar represents MSSA with MRSA associated MLST CCs $(n=75)$ and the light grey bar represents MSSA with MSSA associated MLST CCs $(n=8 I)$. Asterisks denote statistically significant difference $P<0.0$ I (a) and statistically significant difference of individual CCs versus all other associated MLST CCs (b) $P<0.01$.

film formation, namely 5\%, 15\%, 45\% and $90 \%$ at $0 \%$, $0.1 \%, 0.25$ and $0.5 \%$ glucose, respectively.

\section{Distribution of agr types}

Clonal lineages MLST CC7, CC8, CC22, CC25 and CC45 harbored agr-I, all CC5, CC12 and CC15 were character-

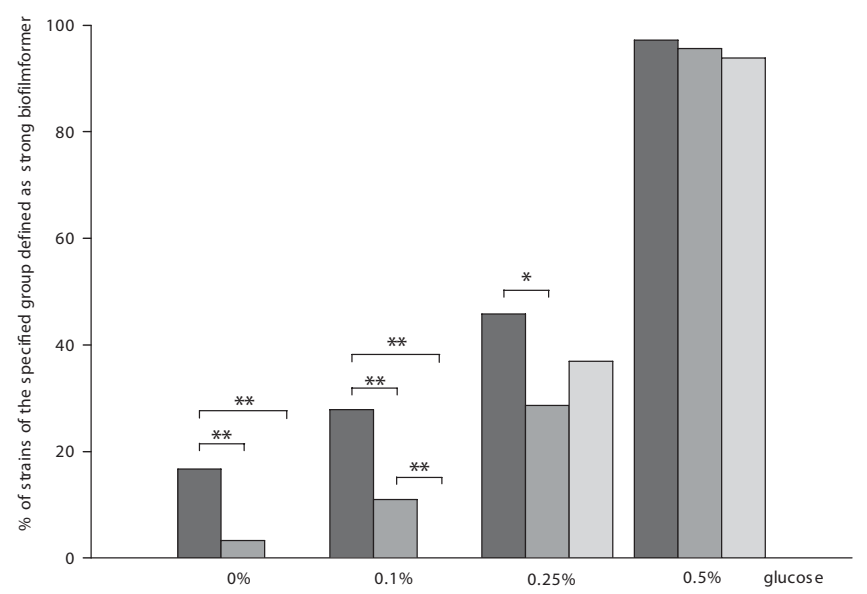

Figure 2

Quantification of strong biofilm formation in MSSA and MRSA. Quantification of strains of the specified group defined as strong biofilm former at different glucose concentrations. Black bars represent MRSA, dark grey bars represent MSSA with MRSA associated MLST CCs and light grey bars represent MSSA with MSSA associated MLST CCs. Asterisks denote statistically significant difference, $\left({ }^{*}\right) P<$ 0.05 and $(* *) P<0.01$.

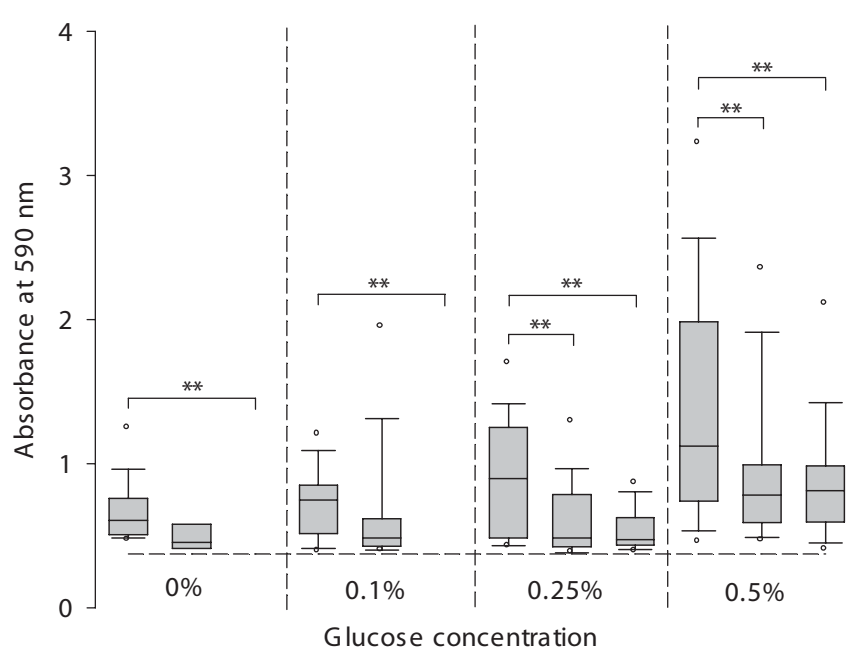

Figure 3

Biomass quantification of MSSA and MRSA. Absorbance $\left(A_{590}\right)$ of the crystal violet stained biofilm matrix for strong biofilm formers (with $A_{590}$ above the threshold value of 0.374 , represented by the horizontal dashed line) at different glucose concentrations. Boxplots at the left show MRSA, in the middle MSSA with MRSA associated MLST CCs and at the right MSSA with MSSA associated MLST CCs. The lower and higher boundary of the box indicates the $25^{\text {th }}$ and $75^{\text {th }}$ percentile, respectively. The line within the box marks the median. Whiskers above and below the box indicate the $90^{\text {th }}$ and $10^{\text {th }}$ percentiles. Open circles indicate the $95^{\text {th }}$ and $5^{\text {th }}$ percentiles. Asterisks denote statistically significant difference, $(*) P<0.05$ and $(* *) P<0.01$.

ized by agr-II, while all CC1 and CC30 were detected as agr-III. Furthermore, CC121 isolates carried agr-IV (Table 1). No consistent relationship was found between agr genotype and the ability to produce biofilm.

\section{Discussion}

In vitro quantification of biofilm formation in distinct clonal lineages of $S$. aureus was performed to investigate whether there were differences in the capacity to form fully established biofilms. This study revealed that at $0.1 \%$ glucose, enhanced biofilm formation of $S$. aureus was strongly associated with MLST CC8 and observed in $60 \%$ of these isolates, while it varied between $0-7 \%$ for the other clonal lineages tested.

A higher percentage of MSSA (14\%) than MRSA (0\%) was found positive for slime producing ability, in concordance to the more important role of PIA/PNAG in MSSA than in MRSA biofilm development [8]. Addition of sucrose to CRA did not influence slime formation, suggesting that slime formation was carbohydrates independent. The results were consistent with previous findings in MRSA and MSSA isolates of O'Neill et al. In MSSA isolates increased ica expression and PIA/PNAG production (as 

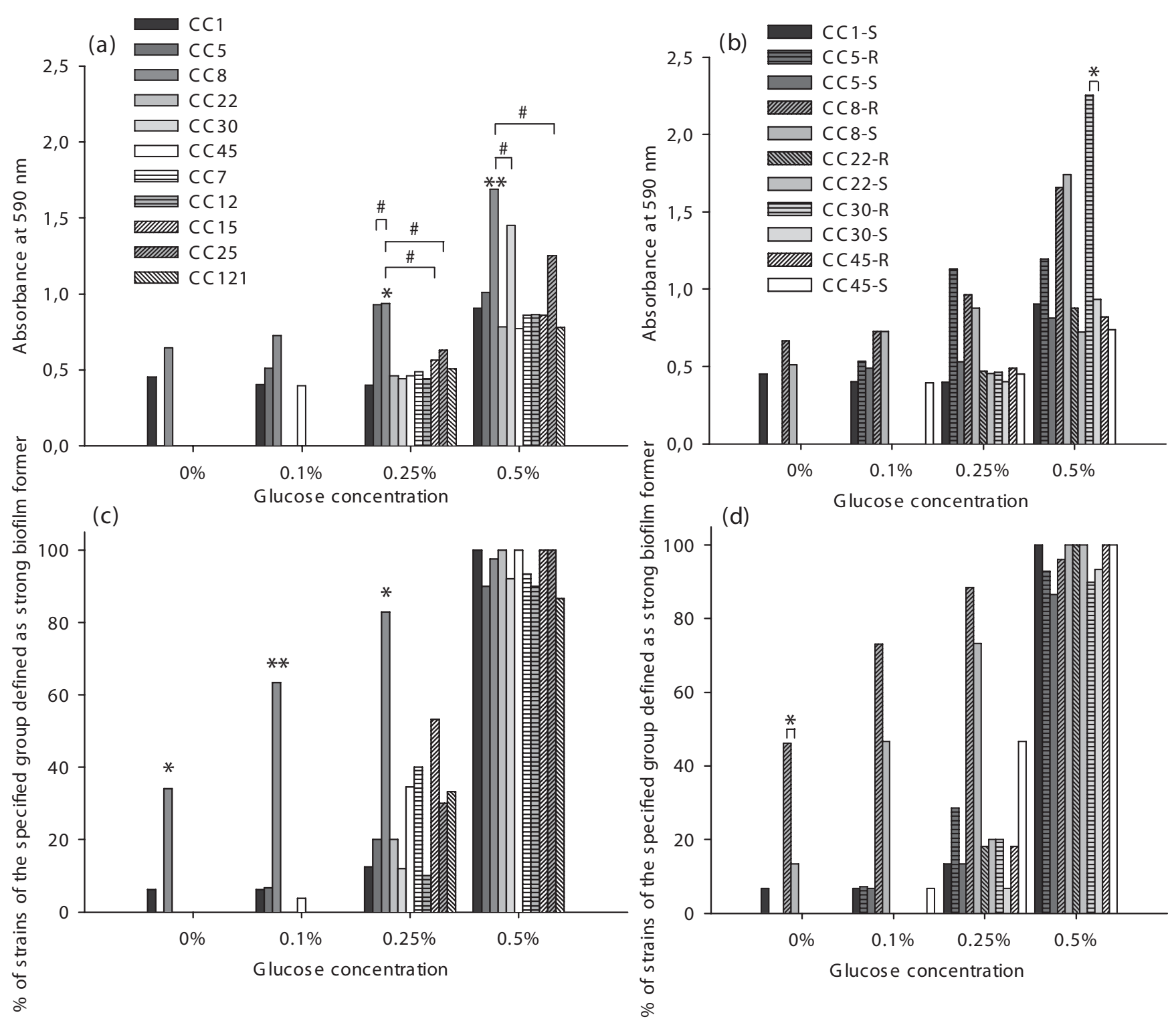

Figure 4

Biomass formation related to the genetic background of $\mathbf{S}$. aureus. Absorbance $\left(A_{590}\right)$ of the crystal violet stained biofilm matrix of strong biofilm forming S. aureus strains in relation to different associated MLST CCs (a) and of strong biofilm forming strains associated with MLST CCI, CC5, CC8, CC22, CC30 and CC45 (b). R in the legend represents MRSA and S represents MSSA. Quantification of strains of the specified genetic background defined as strong biofilm former at different glucose concentrations, (c) and (d). Asterisks denote statistically significant difference, (b) and (d), and statistical significant difference of individual CCs versus all other associated MLST CCs, (a) and (c), except \#, (*) $P<0.05$ and $(* *) P<0.0$ I.

determined with PIA/PNAG immunoblot) was correlated with $4 \% \mathrm{NaCl}$-induced biofilm formation, but not with glucose-induced biofilm production [8]. In addition, in MRSA, ica operon transcription was more potently activated by $\mathrm{NaCl}$ than by glucose, but did not result in PIA/ PNAG formation [8]. Since it has recently been suggested that, in general, PIA/PNAG is a minor matrix component of $S$. aureus biofilms $[5,9]$, and thus possibly hardly detectable by CRA screening, a low prevalence of slime producing strains was expected. Knobloch et al. and Mathur et al. reported a positive CRA assay result in only $4-5 \%$ of the $S$. aureus strains tested, in relative accordance with the results of this study, while Grinholc et al. mentioned $47 \%$ and $69 \%$ for MRSA and MSSA, respectively [16-18]. Jain et al. reported differences between blood stream isolates and commensal $S$. aureus isolates with 

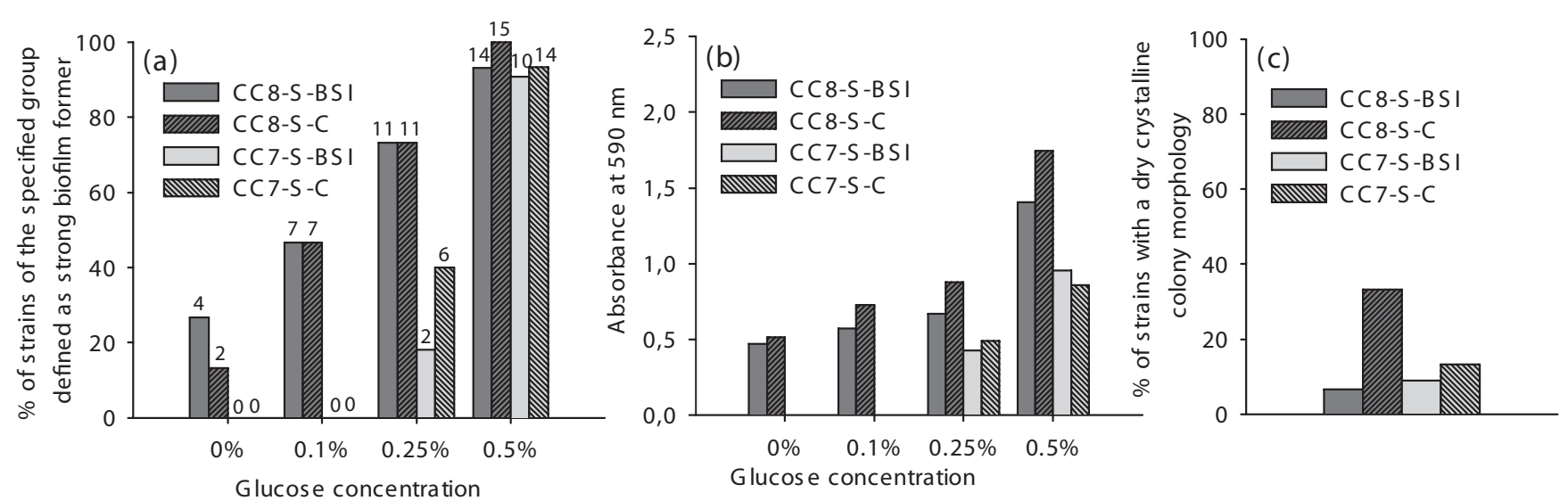

Figure 5

Biofilm formation in S. aureus isolates of bloodstream infections and commensal origin. Biofilm formation between S. aureus isolates of the same clonal lineage from blood stream infections (CC8 $n=15, C C 7 n=1 \mathrm{I})$ and of commensal origin $(\mathrm{CC8} n=15, \mathrm{CC7} n=15)$, no significant differences were found (a). S in the legend represents MSSA, BSI represents bloodstream isolates and $C$ represents commensal isolates. Number on each bar refers to number of isolates. $A b s o r b a n c e ~\left(A_{590}\right)$ of the crystal violet stained biofilm matrix of strong biofilm formers at different glucose concentrations (b). CRA screening for colonies with a dry crystalline morphology (c).

regard to positive CRA screening, $75 \%$ and $20 \%$, respectively [20]. The variations could be due to differences in genetic backgrounds of the strains used, or to differences in interpretation of the colonies. The definition of slimeforming strains used by Grinholc et al. and Jain et al. was based on the color of the colonies and not on the morphology. Furthermore, they both found a high consistency ( $96 \%$ and $91 \%$, respectively) between CRA screening and biofilm biomass crystal violet staining $[17,20]$. In contrast, both in this study, as well in the studies by Knobloch et al., Rode et al., and Mathur et al. [16,18,21], no correlation was found between slime producing MRSA and MSSA isolates and an enhanced tendency to form large amounts of biomass. These studies strongly suggest that CRA screening forms no alternative for crystal violet staining to detect biofilm formation. Probably, the cell to cell adhesion, stimulated by the formation of PIA/PNAG, is less efficient than the expression of surface adhesins, in their contribution to produce more biomass.
As described before, the agr genotypes were strictly associated with the clonal lineages $[22,23]$. However, exceptions have been observed [24-27] which might be due to interstrain recombination and intrastrain rearrangements [28]. The association between agr genotypes and the genetic background explains the absence of a relationship between the enhanced ability to form biofilm and specific agr genotype(s). Although, there was a tendency that the $a g r$-I genotype was associated with an enhanced capacity to form strong biofilms (data not shown), this was a reflection of the biofilm-forming capacity of strains associated with MLST CC8. In contrast to our results, Cafiso et al. described a link between agr-II genotype and the capacity to form strong biofilms, since all strains with $a g r$-II genotype were associated with strong biofilm formation at $0.25 \%$ glucose. However, the genetic background was not taken into consideration [29]. Our findings revealed that strains associated with MLST CC5, CC12 and CC15 (all harboring $a g r$-II) were classified as strong biofilm formers in only $21 \%, 9 \%$ and $53 \%$ of the cases at $0.25 \%$

Table 2: Correlation between slime formation (Congo red agar screening) and development of biofilm biomass (crystal violet staining).

\begin{tabular}{ccccccccc}
\hline $\begin{array}{c}\text { Glucose } \\
(\%)\end{array}$ & $\begin{array}{c}\text { Sensitivity } \\
\text { (\%) }\end{array}$ & $\begin{array}{c}\text { Specificity } \\
(\%)\end{array}$ & $\begin{array}{c}\text { PPV } \\
(\%)\end{array}$ & $\begin{array}{c}\text { NPV } \\
(\%)\end{array}$ & $\begin{array}{c}\text { CRA+/CV+ } \\
\text { (\%) }\end{array}$ & $\begin{array}{c}\text { CRA-/CV+ } \\
\text { Number of S. aureus strains }\end{array}$ & $\begin{array}{c}\text { CRA+/CV- } \\
\text { CRA-/CV- }\end{array}$ \\
\hline 0 & 6.3 & 91.0 & 5.0 & 92.8 & 1 & 15 & 19 & 193 \\
0.1 & 9.7 & 91.3 & 15.0 & 86.5 & 3 & 28 & 17 & 180 \\
0.25 & 11.6 & 93.0 & 45.0 & 63.5 & 11 & 76 & 9 & 132 \\
0.5 & 8.3 & 80.0 & 90.0 & 3.9 & 18 & 200 & 2 & 8
\end{tabular}

(PPV) positive predictive value (NPV) negative predictive value (CRA) Congo red agar screening (CV) crystal violet staining 
glucose, respectively. Furthermore, the agr-II genotype encompass diverse strains, not including strains associated with MLST CC8 [22,23].

Biofilm formation was induced by increasing glucose concentrations up to $0.5 \%$ in both MRSA and MSSA isolates, which is entirely consistent with previously reported data [8,21]. However, MRSA produced significantly more biomass than MSSA with MSSA associated MLST CCs, under all tested glucose conditions. Especially strains associated with MLST CC8 contributed to this phenomenon. Furthermore, MSSA with MRSA associated MLST CCs were also capable to produce more biomass than MSSA with MSSA associated MLST CCs at $0.1 \%$ glucose. Variations in biofilm forming capacities in clonal lineages of $S$. aureus could be explained by unique combinations of surfaceassociated and regulatory genes [23] or by different expression levels of genes that regulate the different phases of biofilm formation. Since this study showed that the biofilm formation on polystyrene surfaces was the strongest for the MLST CC8 associated genetic background, further studies with other material or tissue are warranted. Recently, differences in adhesion to human airway epithelial cells have been observed between strains belonging to MLST CC8 and CC5, the latter demonstrating a generally lower adherence in both representatives of MRSA and MSSA [30]. An enhanced ability to adhere and invade these cells have also been shown for MRSA associated with the Brazilian/Hungarian clone, which belongs to MLST CC8 [15], compared to a population of MSSA with an unknown genetic background [31]. Furthermore, strains associated with the same clone were not included among our MLST CC8 isolates, but were previously classified as strong biofilm producers and designated superior in their ability to produce biofilm compared to isolates associated with the Pediatric clone (MLST CC5) [32].

To analyse possible other predisposing factors besides the MLST CC8 associated genetic background, bloodstream and commensal isolates of the same clonal lineage were compared. The biofilm forming capacity between MSSA bloodstream and commensal isolates, associated with MLST CC8 and CC7, was similar and consistent with the findings of Smith et al., who compared the biofilm forming capacity of Scottish clinical S. aureus strains collected from different isolation sites [33]. In contrast, Jain et al. described more frequently strong biofilm formers among S. aureus bloodstream isolates than commensal [20]. A possible explanation might be that all bloodstream isolates came from patients with peripheral intravenous devices, while this was not an inclusion criterion in the study by Smith et al. Peripheral or central line intraluminal colonization might be associated with strains that easily attach to (catheter) surfaces and as a consequence these strains could be dominant in leading to bloodstream infections.

\section{Conclusion}

In summary, the present study reveals that the MLST CC8 associated genetic background was a predisposing factor for strong biofilm formation in vitro, under all tested glucose concentrations, i.e. $0 \%, 0.1 \%, 0.25 \%$ and $0.5 \%$. At physiologic glucose concentration $(0.1 \%), 0-7 \%$ of $S$. aureus from various clonal lineages were defined as strong biofilm former, compared to $60 \%$ for the $S$. aureus associated MLST CC8.

\section{Methods}

\section{Bacterial strains}

S. aureus strains (72 MRSA and 156 MSSA) investigated were isolated during 2005 to 2008 in the Maastricht University Medical Center, a tertiary 715-bed hospital, and originate from surveillance cultures (commensal flora) from individual patients, recovered from nasal swabs. MRSA and/or MSSA strains associated with MLST CC1, CC5, CC8, CC22, CC30, CC45, CC7, CC12, CC15, CC25 and CC121, were randomly selected from our institutional collection (Table 1). All MRSA strains were tested positive for the MRSA-specific mecA gene, by real-time PCR [34]. Additionally, 26 MSSA blood stream isolates from individual patients and associated with either MLST CC8 or CC7 were tested. These isolates were considered invasive.

\section{Characterization of the genetic background}

Typing of the spa locus was carried out as described previously [19]. The spa types were assigned through the Ridom SpaServer http://spaserver.ridom.de and clustered into spa-CCs using the algorithm based upon repeat pattern (BURP) with Ridom StaphType 1.4 using the default settings $[35,36]$. Although, spa typing alone sometimes lacks discriminatory power, due to related spa repeat patterns within different clonal lineages and the emergence of homoplasies among spa sequences [37], it has been shown that spa typing/BURP results are often in agreement with results obtained by MLST $[36,38]$. Therefore, the associated MLST CCs were allocated through the SpaServer. To confirm the association between MLST and spa typing, in combination with BURP, MLST was performed on a representative set of 16 strains of each major spa type and associated MLST CC $[39,40]$.

\section{Phenotypic detection of slime producing ability onto Congo red agar}

MRSA $(n=72)$, MSSA with MRSA associated MLST CCs $(n$ = 75), i.e. CC1, CC5, CC8, CC22, CC30 and CC45, and MSSA with MSSA associated MLST CCs $(n=81)$, i.e. CC7, CC12, CC15, CC25 and CC121, were cultured on Congo red agar (CRA) plates, either consisting of trypticase soy or brain heart infusion agar (both from Becton Dickinson) with $0.8 \mathrm{~g} / \mathrm{L}$ Congo red (Prolabo, Leuven, Belgium) and without or with 5\% sucrose (Merck, Darmstadt, Germany). Colony morphology and color were evaluated 
after incubation at $37^{\circ} \mathrm{C}$ for $24 \mathrm{~h}$. Colonies with a dry crystalline (rough) morphology were considered deviant and slime producing positive [16], smooth round colonies were classified as low-slime producers.

\section{Detection of biofilm biomass with crystal violet staining}

The polystyrene crystal violet adherence assay was carried out as described previously [41], with some modifications. Briefly, overnight cultures in Trypticase Soy Broth (TSB) without dextrose (Becton Dickinson, Le pont de Claix, France) were diluted until $10^{8} \mathrm{CFU} / \mathrm{mL}$ in TSB containing $0 \%, 0.1 \%, 0.25 \%$ and $0.5 \%$ glucose. Individual wells of polystyrene, flat-bottomed 96-well plates (Greiner Bio-One, Frickenhausen, Germany) were filled with $100-\mu \mathrm{L}$ aliquots of the cultures. As a negative control, uninoculated medium was used. S. aureus ATCC 25923 and one clinical $S$. aureus isolate from our collection, known to form fully established biofilms $\left(A_{590}\right.$ values within the highest range and stable) as observed during a pilot experiment, were added to each plate as reference standard [17] and positive control, respectively. After $4 \mathrm{~h}$ of adhesion at $37^{\circ} \mathrm{C}$ on a rocking platform at 25 oscillations $\mathrm{min}^{-1}$, the medium containing non-adhered cells, was replaced by $100 \mu \mathrm{L}$ fresh broth and the plates were further incubated for $24 \mathrm{~h}$. Next, the wells were washed three times with $200 \mu \mathrm{L} 0.9 \% \mathrm{NaCl}$. Biofilms were fixed at $60^{\circ} \mathrm{C}$ during $1 \mathrm{~h}$. Subsequently, $100 \mu \mathrm{l}$ crystal violet solution $(0.3 \% \mathrm{wt} / \mathrm{vol})$ was added to all wells. After $15 \mathrm{~min}$, the excess crystal violet was rinsed off by placing the plates under running tap water. Finally, after drying the plates, bound crystal violet was released by adding $100 \mu \mathrm{l} 70 \%$ (vol/vol) ethanol with $10 \%$ isopropyl alcohol (vol/vol). Absorbance was measured spectrophotometrically at 590 $\mathrm{nm}\left(A_{590}\right)$ and was proportional to biofilm biomass. All assays were performed in triplicate, and repeated on three occasions. The intra- and interday coefficients of variation for the assay were $14 \%$ and $23 \%$, respectively. To obtain a threshold $A_{590}$ value for which strong biofilm formation commences, the $A_{590}$ values of all strains at the different glucose concentrations were sorted in ascending order and divided into quartiles. The distribution of $A_{590}$ values in the lower three quartiles was similar at glucose concentrations of $0 \%, 0.1 \%$ and $0.25 \%$ and therefore used to determine the cut-off value (two standard deviations above the mean $A_{590}$ value). The threshold $A_{590}$ value was 0.374 . Bacteria with $A_{590}$ values above this value were considered strong biofilm formers.

\section{Determination of the agr type}

The agr types were determined by a real-time multiplex PCR assay, as described previously [42].

\section{Statistical analysis}

SPSS version 15.0 (SPSS Inc., Chicago, IL, USA) was used for statistical analyses. Chi-square analysis was used for comparison of the prevalence of strong biofilm formation or slime formation between the specified groups. MannWhitney $U$ analysis was used to compare the $A_{590}$ values between groups of strong biofilm formers. A $P$ value of $<$ 0.05 was considered to be statistically significant.

\section{Competing interests}

The authors declare that they have no competing interests.

\section{Authors' contributions}

SC carried out the biofilm measurement experiments and performed MLST, collected data and drafted the manuscript. RHD carried out the spa typing/BURP and participated in the design of the study. MLLB determined the agr types by a real-time multiplex PCR, helped with the statistical analysis and helped to write the manuscript. $\mathrm{PB}$ revised the manuscript critically. $\mathrm{CN}$ revised the manuscript critically. EES conceived of the study and participated in its design and coordination and helped to draft the manuscript. All authors read and approved the final manuscript

\section{Acknowledgements}

We thank L. Sheriff and M.I.A. Rijnders for technical assistance.

Funding

No financial support was received

\section{References}

I. Patel R: Biofilms and antimicrobial resistance. Clin Orthop Relat Res 2005:41-47.

2. Leid JG, Shirtliff ME, Costerton JW, Stoodley AP: Human leukocytes adhere to, penetrate, and respond to Staphylococcus aureus biofilms. Infect Immun 2002, 70(I I):6339-6345.

3. Costerton JW, Stewart PS, Greenberg EP: Bacterial biofilms: a common cause of persistent infections. Science 1999, 284(54 | 8): I 3 | $18-1322$.

4. Foster TJ, Hook M: Surface protein adhesins of Staphylococcus aureus. Trends Microbiol 1998, 6(1 2):484-488.

5. Boles BR, Horswill AR: Agr-mediated dispersal of Staphylococcus aureus biofilms. PLoS Pathog 2008, 4(4): 1000052.

6. Vuong C, Saenz HL, Gotz F, Otto M: Impact of the agr quorumsensing system on adherence to polystyrene in Staphylococcus aureus. J Infect Dis 2000, I 82(6): 1688- 1693.

7. O'Gara JP: ica and beyond: biofilm mechanisms and regulation in Staphylococcus epidermidis and Staphylococcus aureus. FEMS Microbiol Lett 2007, 270(2): 179-188.

8. O'Neill E, Pozzi C, Houston P, Smyth D, Humphreys H, Robinson DA, O'Gara JP: Association between methicillin susceptibility and biofilm regulation in Staphylococcus aureus isolates from device-related infections. J Clin Microbiol 2007, 45(5): I 379-I 388.

9. Izano EA, Amarante MA, Kher WB, Kaplan JB: Differential roles of poly-N-acetylglucosamine surface polysaccharide and extracellular DNA in Staphylococcus aureus and Staphylococcus epidermidis biofilms. Appl Environ Microbiol 2008, 74(2):470-476.

10. Regassa LB, Novick RP, Betley MJ: Glucose and nonmaintained $\mathrm{pH}$ decrease expression of the accessory gene regulator (agr) in Staphylococcus aureus. Infect Immun 1992, 60(8):338I-3388.

II. O'Neill E, Pozzi C, Houston P, Humphreys H, Robinson DA, Loughman A, Foster TJ, O'Gara JP: A novel Staphylococcus aureus biofilm phenotype mediated by the fibronectin-binding proteins, FnBPA and FnBPB. J Bacteriol 2008, I90( I I):3835-3850. 
12. Guyton AC, Hall JE, eds: Textbook of medical physiology. 10th edition. Philadelphia: W.B. Saunders company; $200 I$.

13. Deurenberg RH, Vink C, Kalenic S, Friedrich AW, Bruggeman CA, Stobberingh EE: The molecular evolution of methicillin-resistant Staphylococcus aureus. Clin Microbiol Infect 2007, I3(3):222-235.

14. Noto MJ, Kreiswirth BN, Monk AB, Archer GL: Gene acquisition at the insertion site for $\mathrm{SCCmec}$, the genomic island conferring methicillin resistance in Staphylococcus aureus. J Bacteriol 2008, 190(4): | 276-| 283.

15. Deurenberg RH, Stobberingh EE: The evolution of Staphylococcus aureus. Infect Genet Evol 2008, 8(6):747-763.

16. Knobloch JK, Horstkotte MA, Rohde H, Mack D: Evaluation of different detection methods of biofilm formation in Staphylococcus aureus. Med Microbiol Immunol 2002, 191(2): I0I-106.

17. Grinholc M, Wegrzyn G, Kurlenda J: Evaluation of biofilm production and prevalence of the icaD gene in methicillin-resistant and methicillin-susceptible Staphylococcus aureus strains isolated from patients with nosocomial infections and carriers. FEMS Immunol Med Microbiol 2007, 50(3):375-379.

18. Mathur T, Singhal S, Khan S, Upadhyay DJ, Fatma T, Rattan A: Detection of biofilm formation among the clinical isolates of Staphylococci: an evaluation of three different screening methods. Indian J Med Microbiol 2006, 24(I):25-29.

19. Nulens E, Stobberingh EE, van Dessel H, Sebastian S, van Tiel FH, Beisser PS, Deurenberg RH: Molecular characterization of Staphylococcus aureus bloodstream isolates collected in a Dutch University Hospital between 1999 and 2006. J Clin Microbiol 2008, 46(7):2438-244I.

20. Jain A, Agarwal A: Biofilm production, a marker of pathogenic potential of colonizing and commensal staphylococci. J Microbiol Methods 2009, 76(I):88-92.

21. Rode TM, Langsrud S, Holck A, Moretro T: Different patterns of biofilm formation in Staphylococcus aureus under foodrelated stress conditions. Int J Food Microbiol 2007, I I 6(3):372-383

22. Monecke S, Slickers P, Ehricht R: Assignment of Staphylococcus aureus isolates to clonal complexes based on microarray analysis and pattern recognition. FEMS Immunol Med Microbiol 2008, 53(2):237-25I.

23. Lindsay JA, Moore CE, Day NP, Peacock SJ, Witney AA, Stabler RA, Husain SE, Butcher PD, Hinds J: Microarrays reveal that each of the ten dominant lineages of Staphylococcus aureus has a unique combination of surface-associated and regulatory genes. J Bacteriol 2006, I 88(2):669-676.

24. Holtfreter S, Grumann D, Schmudde M, Nguyen HT, Eichler P, Strommenger B, Kopron K, Kolata J, Giedrys-Kalemba S, Steinmetz I, et al.: Clonal distribution of superantigen genes in clinical Staphylococcus aureus isolates. J Clin Microbiol 2007, 45(8):2669-2680.

25. Luczak-Kadlubowska A, Sulikowska A, Empel J, Piasecka A, Orczykowska M, Kozinska A, Hryniewicz W: Countrywide molecular survey of methicillin-resistant Staphylococcus aureus strains in Poland. J Clin Microbiol 2008, 46(9):2930-2937.

26. Layer F, Ghebremedhin B, Konig W, Konig B: Heterogeneity of methicillin-susceptible Staphylococcus aureus strains at a German University Hospital implicates the circulating-strain pool as a potential source of emerging methicillin-resistant S. aureus clones. J Clin Microbiol 2006, 44(6):2 I79-2। 85

27. Peacock SJ, Moore CE, Justice A, Kantzanou M, Story L, Mackie K, O'Neill G, Day NP: Virulent combinations of adhesin and toxin genes in natural populations of Staphylococcus aureus. Infect Immun 2002, 70(9):4987-4996.

28. Wright JS, Traber KE, Corrigan R, Benson SA, Musser JM, Novick RP. The agr radiation: an early event in the evolution of staphylococci. J Bacteriol 2005, I 87( (16):5585-5594.

29. Cafiso V, Bertuccio T, Santagati M, Demelio V, Spina D, Nicoletti G, Stefani S: agr-Genotyping and transcriptional analysis of biofilm-producing Staphylococcus aureus. FEMS Immunol Med Microbiol 2007, 5 I (I):220-227.

30. Karauzum H, Ferry T, de Bentzmann S, Lina G, Bes M, Vandenesch F, Schmaler M, Berger-Bachi B, Etienne J, Landmann R: Comparison of adhesion and virulence of two predominant hospitalacquired methicillin-resistant Staphylococcus aureus clones and clonal methicillin-susceptible $\mathbf{S}$. aureus isolates. Infect Immun 2008, 76( I I):5I33-5I38.
31. Amaral MM, Coelho LR, Flores RP, Souza RR, Silva-Carvalho MC, Teixeira LA, Ferreira-Carvalho BT, Figueiredo AM: The predominant variant of the Brazilian epidemic clonal complex of methicillin-resistant Staphylococcus aureus has an enhanced ability to produce biofilm and to adhere to and invade airway epithelial cells. J Infect Dis 2005, I 92(5):80 I-8I 0.

32. de Miranda OP, Silva-Carvalho MC, Ribeiro A, Portela F, Cordeiro RP, Caetano N, Vidal CF, Figueiredo AM: Emergence in Brazil of methicillin-resistant Staphylococcus aureus isolates carrying SCCmecIV that are related genetically to the USA800 clone. Clin Microbiol Infect 2007, I3(12): I I65- I 172.

33. Smith K, Perez A, Ramage G, Lappin D, Gemmell CG, Lang S: Biofilm formation by Scottish clinical isolates of Staphylococcus aureus. J Med Microbiol 2008, 57(Pt 8): 1018-1023.

34. Donker GA, Deurenberg RH, Driessen C, Sebastian S, Nys S, Stobberingh EE: The population structure of Staphylococcus aureus among general practice patients from The Netherlands. Clin Microbiol Infect 2009, I 5(2): I37-I 43

35. Friedrich AW, Witte W, Harmsen D, de Lencastre H, Hryniewicz W, Scheres J, Westh H: SeqNet.org: a European laboratory network for sequence-based typing of microbial pathogens. Euro Surveill 2006, I I(I):E060I I 2060 I I4.

36. Strommenger B, Kettlitz C, Weniger T, Harmsen D, Friedrich AW, Witte W: Assignment of Staphylococcus isolates to groups by spa typing, Smal macrorestriction analysis, and multilocus sequence typing. I Clin Microbiol 2006, 44(7):2533-2540.

37. Nubel U, Roumagnac P, Feldkamp M, Song JH, Ko KS, Huang YC, Coombs G, Ip M, Westh H, Skov R, et al.: Frequent emergence and limited geographic dispersal of methicillin-resistant Staphylococcus aureus. Proc Natl Acad Sci USA 2008, 105(37): $14|30-14| 35$

38. Ruppitsch W, Indra A, Stoger A, Mayer B, Stadlbauer S, Wewalka G, Allerberger $\mathrm{F}$ : Classifying spa types in complexes improves interpretation of typing results for methicillin-resistant Staphylococcus aureus. J Clin Microbiol 2006, 44(7):2442-2448.

39. Deurenberg RH, Vink C, Oudhuis G], Mooij JE, Driessen C, Coppens G, Craeghs J, De Brauwer E, Lemmen S, Wagenvoort H, et al.: Different clonal complexes of methicillin-resistant Staphylococcus aureus are disseminated in the Euregio Meuse-Rhine region. Antimicrob Agents Chemother 2005, 49(10):4263-427I.

40. Enright MC, Day NP, Davies CE, Peacock SJ, Spratt BG: Multilocus sequence typing for characterization of methicillin-resistant and methicillin-susceptible clones of Staphylococcus aureus. J Clin Microbiol 2000, 38(3): I008-10I5.

4I. Peeters E, Nelis HJ, Coenye T: Comparison of multiple methods for quantification of microbial biofilms grown in microtiter plates. J Microbiol Methods 2008, 72(2): I57-165.

42. Francois $P$, Koessler $T$, Huyghe $A$, Harbarth $S$, Bento M, Lew D, Etienne J, Pittet D, Schrenzel J: Rapid Staphylococcus aureus agr type determination by a novel multiplex real-time quantitative PCR assay. J Clin Microbiol 2006, 44(5): | 892-I895.

Publish with Biomed Central and every scientist can read your work free of charge

"BioMed Central will be the most significant development for disseminating the results of biomedical research in our lifetime. "

Sir Paul Nurse, Cancer Research UK

Your research papers will be:

- available free of charge to the entire biomedical community

- peer reviewed and published immediately upon acceptance

- cited in PubMed and archived on PubMed Central

- yours - you keep the copyright 